\title{
Avaliação do teor de Vitamina C em morangos de diferentes cultivares em sistemas de cultivo distintos no municipio de Bom Princípio/RS
}

\author{
Vitamin C content of strawberries evaluation of different cultivars in different \\ cropping systems in Bom Princípio /RS
}

\author{
Cristiane Inês Musa ${ }^{1}$, Bárbara Weber ${ }^{2}$, Helen Cristina Gonzatti ${ }^{3}$, Cecília Brasil Biguelini' ${ }^{4}$, \\ Claucia Fernanda Volken de Souza ${ }^{5}$ e Eniz Conceição Oliveira ${ }^{6}$ \\ ${ }^{1}$ Centro Universitário Univates - Instituto Federal de Educação, \\ Ciência e Tecnologia do Rio Grande do Sul - IFRS/Câmpus Feliz, RS, Brasil

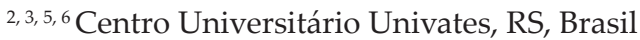 \\ ${ }^{4}$ Centro Universitário Univates - Instituto Federal de Educação, \\ Ciência e Tecnologia do Rio Grande do Sul - IFRS/, RS, Brasil
}

\begin{abstract}
Resumo
O morango é um pseudofruto muito apreciado pelo seu sabor, aroma e propriedades nutricionais, contendo importante fonte de vitamina C. Tal vitamina é fundamental para a saúde humana, sendo que seu consumo está associado à redução do risco de diversas doenças. O presente artigo visa avaliar o teor da vitamina $C$ em morangos, analisando a influência dos cultivares Festival e San Andreas nos sistemas de cultivo convencional e orgânico, em substrato, no município de Bom Princípio, Rio Grande do Sul. O teor de vitamina C foi avaliado através de cromatografia de alta eficiência em comparação a uma curva padrão. Os teores de vitamina $C$ encontrados nos cultivares Festival e San Andreas foram superiores no sistema convencional em relação ao sistema orgânico, isto é, 34,85 e 69,31 mg/100 g ao passo de 16,62 e 42,19 mg/100 g, respectivamente.
\end{abstract}

Palavras-chave: Ácido ascórbico. Morangueiro. Cultivo em substrato

\begin{abstract}
The strawberry is a pseudo much appreciated for its taste, aroma and nutritional properties, containing important source of vitamin C. This vitamin is essential for human health, and its consumption is associated with reduced risk of several diseases. This article aims to assess the vitamin $C$ content in strawberries, analyzing the influence of the Festival and San Andreas cultivars in conventional and organic farming systems, substrate, in Bom Pricipio, Rio Grande do Sul. The vitamin C content was assessed by high performance liquid chromatography in comparison to a standard curve. Vitamin C content found in the Festival and San Andreas cultivars were higher than in the conventional system with respect to the organic system, i.e., 34.85 and $69.31 \mathrm{mg} / 100 \mathrm{~g}$ at step 10016.62 and 42.19 $m g / 100 \mathrm{~g}$, respectively.
\end{abstract}

Keywords: Ascorbic acid. Strawberry. Substrate cultivation 


\section{Introdução}

A $s$ frutas apresentam diversas qualidades nutritivas. Neste sentido, estudos epidemiológicos têm demonstrado que uma dieta rica em vegetais e frutas está associada com o aumento da expectativa de vida, trazendo muitos benefícios à saúde (Rice-Evans; Miller, 1995).

Salienta-se que o morango (Fragaria $X$ ananassa Duch), pertencente à família das Rosáceas é uma planta herbácea, perene e com caule semi-subterrâneo. É sensível à baixa umidade, falta de água, intensidade e duração da luz e alta temperatura. A parte comestível é constituída por um receptáculo suculento e carnoso, formando um pseudofruto (Oshita; Jardim, 2012; Sanhueza et al., 2005).

Cabe mencionar que o cultivo do morangueiro apresenta um papel econômico e social preponderante na região do Vale do Caí no Rio Grande do Sul (RS), especialmente, no município de Bom Princípio. Este município é conhecido nacionalmente pela sua fruta símbolo, o morango (Bom Princípio, 2014).

Aliado a este importante fato, pode-se dizer, também, que o morango possui várias qualidades nutricionais, apresentando micro e macro nutrientes, tendo baixas calorias, sendo fonte de fibras solúveis e minerais (cálcio, ferro, fósforo e potássio) e de vitaminas, dentre elas, A, $B$, ácido fólico $\left(\mathrm{B}_{9}\right)$, e vitamina $\mathrm{C}$ (ácido ascórbico), entre outros compostos (Núcleo de Estudos e Pesquisas em Alimentação, 2011).

Destes compostos, destaca-se a vitamina C, que de acordo com Silva e Cozzolino (2007), tal vitamina está associada à redução do risco de certas doenças. Devido ao seu potencial antioxidante e sua atuação como sequestrante de radicais livres, ela pode ter um possível efeito anticarcinogênico, destoxificando carcinógenos e bloqueando o processo do câncer.

A ingestão de vitamina $C$ está associada, também, com a redução de doenças cardiovasculares prevenindo a oxidação lipídica. Administrada juntamente com a vitamina E e a glutationa, diminui o estresse oxidativo em indivíduos com diabete mellitus, prevenindo a aterosclerose. Outro efeito conhecido é a atuação contra resfriados comuns. Estudos apresentam que o consumo de vitamina $C$ pode diminuir o período da doença e aliviar os sintomas (Silva; Cozzolino, 2007).

A vitamina $C$ ou também denominada ácido ascórbico é sintetizada por plantas e quase todos os animais mamíferos (com exceção dos humanos, primatas, alguns roedores e pássaros). Para os que não a sintetizam, esta vitamina deve ser adquirida na dieta, sendo essencial para a saúde humana, pois sua deficiência pode ocasionar uma doença chamada de escorbuto. Além disso, é muito utilizada na indústria de alimentos como aditivo (Coultate, 2004).

Diante da importância desta vitamina para a saúde humana, o objetivo do trabalho é quantificar o teor de vitamina $\mathrm{C}$ em morangos de diferentes cultivares em sistemas de cultivo distintos no município de Bom Princípio, RS.

\section{Materiais e Métodos}

Os cultivares Festival e San Andreas foram cultivados em sistemas de cultivo convencional e orgânico em substrato. $\mathrm{O}$ trabalho foi realizado em duas propriedades rurais de Bom Princípio/RS, sendo que as coordenadas geográficas das propriedades onde foram coletados os morangos do cultivo convencional em substrato referemse a $-29^{\circ} 29^{\prime} 59,54^{\prime \prime}$ S; $-51^{\circ} 19^{\prime} 52,03^{\prime \prime}$ O e do cultivo orgânico em substrato são: $-29^{\circ} 28^{\prime} 20,15^{\prime \prime}$ S; $-51^{\circ} 21^{\prime} 58,84^{\prime \prime} \mathrm{O}$.

Foi realizado o levantamento da temperatura e da umidade nas duas propriedades rurais pesquisadas, durante 30 dias anteriores à coleta dos morangos, utilizando termo-higromômetro digital marca Incoterm modelo 7666.02.0.00 (Brasil), sendo que todos os registros foram feitos entre $11 \mathrm{~h}$ e $12 \mathrm{~h}$.

A coleta dos morangos foi feita no mês de setembro de 2014 e realizou-se a partir de um plano de amostragem. Tal plano se constituiu em cinco pontos predeterminados, sendo coletados $200 \mathrm{~g}$ no primeiro metro da fileira do plantio, sexto, décimo segundo, décimo oitavo e vigésimo quarto metros, identificados como A, B, C, D e E (Figura 1). Como cada propriedade possui quatro fileiras de 24 $\mathrm{m}$ de substrato de cada cultivar pesquisado, em cada ponto amostrado foram coletados $800 \mathrm{~g}$ de morango.

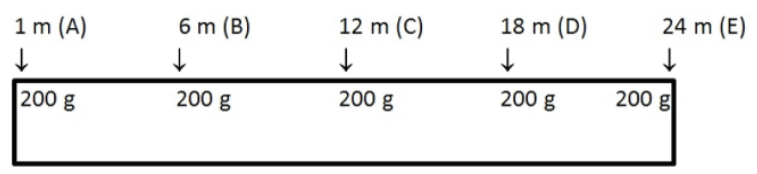

Figura 1: Plano de amostragem da coleta de morangos dos cultivares Festival e San Andreas dos sistemas de cultivo convencional e orgânico, em substrato, no município de Bom Princípio

Foram selecionados morangos segundo o aspecto e o estádio de amadurecimento, ou seja, utilizaram-se morangos que apresentavam coloração 100\% avermelhada. Após, para a preparação da amostra, retirou-se cuidadosamente e manualmente, o cálice juntamente com as sépalas. Posteriormente, os frutos foram lavados com água deionizada e secados com papel absorvente. Triturou-se em um multiprocessador marca Philco, modelo All in one (China). Ressalta-se que a análise de vitamina $C$ foi realizada na data da coleta.

A quantificação da vitamina $C$ foi determinada através do método adaptado de Davey, Bauw e Montagu (1996) e Cruz-Rus et al. (2011), usando ácido metafosfórico $2 \%$ e EDTA 2 mM sob refrigeração para a extração. Salientase que sua quantificação foi feita através de sistema de cromatografia líquida de alta eficiência (CLAE), marca Agilent (Estados Unidos), equipado com detector UV, 
a 254nm, com fase móvel de 0,1 M de fosfato de sódio monobásico e 0,2 mM de EDTA, em fluxo de 0,6 $\mathrm{mL} \mathrm{min}^{-1}$ e coluna Zorbax eclipse plus C18 analytical 4,6x150 mm. O teor de ácido ascórbico presente nos morangos foi calculado a partir dos valores obtidos em curva padrão.

As análises tanto dos substratos quanto das soluções nutritivas foramanalisadas pelos laboratórios da Universidade do Rio Grande do Sul (UFRGS), a saber, Laboratório de Análises de Substratos para Plantas e Laboratório de Solos, respectivamente.

Os substratos foram analisados seguindo a Instrução Normativa da Secretaria de Defesa Agropecuária n. 17, de 21 de maio de 2007 (Brasil, 2007), utilizando Condutivímetro Digimed (Brasil), modelo DM 32, para a análise de condutividade. Já, na análise das soluções nutritivas, foi utilizada condutivimetria com Condutivímetro Digimed (Brasil), modelo DM 32 e Kjeldahl para nitrogênio total.

Os resultados foram analisados estatisticamente através do software SPSS 21 e o nível de significância utilizado foi de $5 \%$.

\section{Resultados e Discussões}

O morango apresenta importante teor de Vitamina C. Contudo, segundo Lee e Kader (2000) e Silva e Cozzolino (2007), esta quantidade pode variar conforme a variedade, o sistema de cultivo, maturação do fruto, condições climáticas pré-colheita e manejo pós-colheita.

Neste sentido, Amaro (2005) e Rocha et al. (2008) destacam, também, que o cultivar pode interferir no teor de vitamina C. Para complementar, Olsson (2004) reitera que entre os cultivares, pode haver variação na concentração de ácido ascórbico de duas a três vezes. Assim, é possível observar que nos resultados encontrados houve uma diferença significativa no teor da vitamina nos dois cultivares pesquisados e entre os distintos sistemas de cultivo (Tabela 1$)$.
Os cultivares Festival e San Andreas apresentaram diferença significativa entre convencional e orgânico (pvalor $<0,001$ ) a um nível de significância de 5\%.

A média dos resultados do teor de vitamina $C$ no sistema convencional e orgânico, em substrato, são 34,85 e de 16,62 mg/100 g, respectivamente, para o cultivar Festival e 69,31 e 42,19 mg/100 g, respectivamente, para San Andreas. A literatura apresenta dados relativos à vitamina C com um valor médio de $60 \mathrm{mg} / 100 \mathrm{~g}$ de fruta (Penteado; Rios, 2003).

No sistema convencional, as diferenças percentuais para o teor de vitamina C foram de $109,7 \%$ e $64,3 \%$ superiores ao sistema orgânico nos cultivares Festival e San Andreas, respectivamente.

Lee e Kader (2000) ressaltam que perdas de vitamina $C$ podem ocorrer devido a sua sensibilidade quanto a condições de baixa umidade.

Diante disso, a média de umidade e temperatura dos 30 dias anteriores à coleta das amostras na propriedade no qual os cultivares Festival e San Andreas no sistema de cultivo orgânico foram coletados, foi de 52\% e a temperatura média de $23^{\circ} \mathrm{C}$. Já, na propriedade no qual houve a coleta dos cultivares Festival e San Andreas no sistema de cultivo convencional, a média da umidade foi de $64 \%$ e a temperatura média de $25,5^{\circ} \mathrm{C}$.

Talvez, um dos fatores que possa ter contribuído para que os teores de vitamina $\mathrm{C}$ dos cultivares do sistema orgânico em substrato sejam inferiores aos cultivares do sistema convencional em substrato (Tabela 1) esteja relacionado com a diferença de $12 \%$ na umidade no mesmo período, ou seja, umidade média de $52 \%$ para o sistema orgânico e $64 \%$ para o sistema convencional.

A pesquisa realizada por Cardoso et al. (2011) avaliou o teor de vitamina $\mathrm{C}$ em morangos do cultivar Oso Grande, oriundo de Atibaia, São Paulo, cultivados em sistema convencional e orgânico, em solo. O teor médio encontrado foi significativamente maior no sistema convencional em comparação ao cultivo orgânico $(p<0,05)$, isto é, $42,45 \mathrm{mg}$ e $30,74 \mathrm{mg} / 100 \mathrm{~g}$ fruta, respectivamente.

Tabela 1 - Teores de vitamina C (mg/100 g) encontrados nos cultivares Festival e San Andreas cultivados nos sistemas convencional e orgânico, em substrato, no município de Bom Princípio

\begin{tabular}{lllll}
\hline \multicolumn{5}{c}{ Vitamina C (mg/100 g) } \\
\hline & \multicolumn{3}{c}{ Festival } & San Andreas \\
\cline { 2 - 5 } & Orgânico & Convencional & Orgânico & Convencional \\
\hline $\mathrm{A}$ & $22,80 \pm 0,07^{\mathrm{c}}$ & $34,79 \pm 1,44^{\mathrm{cb}}$ & $40,44 \pm 0,45^{\mathrm{a}}$ & $68,59 \pm 0,54^{\mathrm{a}}$ \\
$\mathrm{B}$ & $16,80 \pm 0,15^{\mathrm{b}}$ & $39,13 \pm 0,92^{\mathrm{d}}$ & $42,50 \pm 0,20^{\mathrm{b}}$ & $72,49 \pm 0,35^{\mathrm{b}}$ \\
$\mathrm{C}$ & $17,58 \pm 1,73^{\mathrm{b}}$ & $31,92 \pm 0,88^{\mathrm{a}}$ & $45,36 \pm 0,77^{\mathrm{c}}$ & $68,21 \pm 1,59^{\mathrm{a}}$ \\
$\mathrm{D}$ & $12,59 \pm 0,56^{\mathrm{a}}$ & $32,55 \pm 0,55^{\mathrm{ab}}$ & $42,92 \pm 0,42^{\mathrm{b}}$ & $66,50 \pm 0,40^{\mathrm{a}}$ \\
$\mathrm{E}$ & $13,35 \pm 0,62^{\mathrm{a}}$ & $35,86 \pm 0,44^{\mathrm{c}}$ & $39,74 \pm 0,84^{\mathrm{a}}$ & $70,78 \pm 0,39^{\mathrm{b}}$ \\
\hline Média & $16,62 \pm 3,82^{\mathrm{A}}$ & $34,85 \pm 2,73^{\mathrm{B}}$ & $42,19 \pm 2,380^{\mathrm{C}}$ & $69,31 \pm 2,268^{\mathrm{D}}$ \\
\hline
\end{tabular}

\pm Desvio padrão

Médias seguidas de mesma letra não se diferem significativamente, pelo teste de Tukey, ao nível de 5\% de significância 
Tabela 2 - Quantidades de nitrogênio total $\left(\mathrm{mg} \mathrm{L}^{-1}\right)$ das soluções nutritivas utilizadas no cultivo convencional e orgânico, em substrato, dos cultivares Festival e San Andreas

\begin{tabular}{lllllll}
\hline & \multicolumn{4}{c}{ Solução Nutritiva - Nitrogênio total (mg L $\mathbf{~}^{-1}$} \\
\hline & \multicolumn{4}{c}{ Orgânico } & \multicolumn{3}{c}{ Convencional } \\
\cline { 2 - 7 } & Entrada & Saída & Retido & Entrada & Saída & Retido \\
\hline Festival & 91,0 & 15,0 & 76,0 & 35,0 & 3,0 & 32,0 \\
San Andreas & 91,0 & 17,0 & 74,0 & 35,0 & 1,7 & 33,3 \\
\hline
\end{tabular}

Segundo os autores, uma possível explicação para o resultado refere-se ao tipo de fertilização adotado para agricultura convencional, que consistiu numa quantidade menor de fertilizantes nitrogenados.

Conforme Mozafar (1993) e Leee Kader (2000), a aplicação de níveis mais baixos de fertilizantes nitrogenados tem sido associado com um maior teor de vitamina $C$ em frutas e legumes. Altas doses de fertilizantes nitrogenados fazem com que as folhas das plantas aumentem, cobrindo os frutos, diminuindo a passagem de luz e, consequentemente, reduzindo o acúmulo de vitamina $C$ nas partes sombreadas.

Segundo a análise da solução nutritiva, os teores de nitrogênio foram menores no sistema convencional em relação à solução nutritiva do cultivo orgânico (Tabela 2).

Assim sendo, tais resultados corroboram com o exposto por Cardoso et al. (2011), Lee e Karder (2000) e Mozafar (1993). Isto é, os teores de vitamina C foram maiores nos morangos em que foi aplicado um teor menor de nitrogênio, neste caso, no cultivo convencional. A quantidade de nitrogênio da solução nutritiva na entrada foi de $35 \mathrm{mg} \mathrm{L}^{-1}$ para ambos os cultivares. Já, a solução nutritiva de entrada dos cultivares Festival e San Andreas, cultivados no sistema orgânico, apresentaram teores maiores de nitrogênio, a saber, $91 \mathrm{mg} \mathrm{L}^{-1}$, resultando em teores inferiores de vitamina $C$.

A Tabela 1 apresenta os teores de vitamina $C$ encontrados nos cinco pontos coletados (A, B, C, D e E) dos cultivares Festival e San Andreas nos sistemas de cultivo orgânico e convencional, em substrato. $\mathrm{O}$ teste utilizado para verificar se existe diferença significativa nas amostras em cada uma das combinações foi a análise de variância (ANOVA). Este teste mostrou que existe diferença significativa entre as amostras para as combinações orgânico Festival (pvalor<0,001), orgânico
San Andreas (pvalor $<0,001$ ), convencional Festival (pvalor $<0,001$ ) e convencional San Andreas (pvalor $<0,001$ ).

De acordo com a análise estatística, é possível inferir que a localização dos pontos coletados influenciou nos teores de vitamina $C$ encontrados (Tabela 1 ).

Uma possível explicação para as diferenças significativas nos teores de vitamina $\mathrm{C}$ entre os pontos pode ser justificado através de um importante parâmetro que se deve levar em consideração no cultivo do morangueiro, que é a concentração salina. Conforme Godoi (2008), tal parâmetro está relacionado com a quantidade de sais dissolvidos na água, sendo que a condutividade elétrica (CE) mensura esta concentração. Isto é, quanto maior a concentração de sais dissolvidos, maior a medida da CE.

Neste sentido, torna-se necessário avaliar a CE dos substratos utilizados nos cultivos. Vale mencionar que o substrato usado no cultivar Festival nos dois sistemas de cultivo pesquisados foi casca de arroz carbonizada e húmus de celulose e no cultivar San Andreas, também, nos dois sistemas de cultivo foi a turfa. Os valores da CE para os substratos pesquisados estão demonstrados na Tabela 3.

Dessa maneira, observa-se que tanto o substrato do Festival quanto do San Andreas do sistema convencional apresentaram uma CE superior aos substratos dos cultivares do sistema orgânico.

Pode-se considerar que quanto maior a $\mathrm{CE}$, maior os íons dissolvidos na solução, favorecendo que mais nutrientes sejam conduzidos de forma mais homogênea do ponto A até o ponto E.

Para tanto, de acordo com a Tabela 1, o ponto B do cultivar Festival do sistema convencional diferiu significativamente dos pontos C e D, A e D, A e E, apresentando teor de vitamina C de 39,13 mg/ 100g. Porém, os pontos A e E indicaram teores de 34,79 e 35,86 mg/100

Tabela 3 - Valores de condutividade elétrica $\left(\mathrm{mS} \mathrm{cm}^{-1)}\right.$ obtidos nas análises dos substratos constituídos de casca de arroz + húmus de celulose e turfa nos cultivares Festival e San Andreas, respectivamente

Condutividade elétrica $\left(\mathrm{mS} \mathrm{cm}^{-1}\right)$

\begin{tabular}{|c|c|c|c|}
\hline \multicolumn{2}{|c|}{$\begin{array}{l}\text { Casca de arroz carbonizada + húmus } \\
\text { (Festival) }\end{array}$} & \multicolumn{2}{|c|}{$\begin{array}{c}\text { Turfa } \\
\text { (San Andreas) }\end{array}$} \\
\hline Convencional & Orgânico & Convencional & Orgânico \\
\hline 0,23 & 0,10 & 0,29 & 0,19 \\
\hline
\end{tabular}


g, respectivamente. No cultivar San Andreas do sistema convencional, os pontos A, C e D diferiram significativamente dos pontos B e E. Porém, estes dois últimos pontos apresentaram maiores teores de vitamina $\mathrm{C}(72,49$ e 70,78 mg/100 g, respectivamente). Portanto, as medidas superiores de CE relatadas, propiciaram uma condução uniforme dos nutrientes, de modo que os pontos E dos dois cultivares mencionados, apresentaram maiores teores de vitamina $\mathrm{C}$.

Entretanto, os cultivares Festival e San Andreas cultivados no sistema orgânico, indicaram valores de CE inferiores ao sistema convencional, a saber, 0,10 e 0,19 mS $\mathrm{cm}-1$, respectivamente. Conforme os valores de vitamina C obtidos nos pontos, pode-se dizer que o ponto A do cultivar Festival diferiu significativamente, de forma isolada, dos pontos B e C, D e E, com teor de vitamina $\mathrm{C}$ de $22,80 \mathrm{mg} / 100 \mathrm{~g}$, sendo que quanto mais distante de A, menores os teores de vitamina C (Tabela 1). Já, o ponto $C$ do cultivar San Andreas diferiu isoladamente dos pontos A e E, B e D, apresentando o maior teor de vitamina C de $46,36 \mathrm{mg} / 100 \mathrm{~g}$. Neste cultivar, os pontos centrais apresentaram teores superiores aos pontos periféricos, podendo-se inferir que a CE teve uma melhor condução de nutrientes até o centro.

A pesquisa desenvolvida por Portela, Peil e Rombaldi (2012) avaliaram o efeito da concentração da solução nutritiva sobre as características dos compostos fitoquímicos encontrados em morangos. Neste caso, os pesquisadores encontraram teores de vitamina $C$ superiores quando houve elevação da concentração salina da solução nutritiva.

Tal fato também ocorreu na presente pesquisa, pois os cultivares Festival e San Andreas cultivados no sistema convencional em substrato, apresentaram maiores teores de vitamina C (Tabela 1) em relação aos mesmos cultivares no sistema orgânico, sendo que as medidas de CE de saída das soluções nutritivas dos cultivares Festival e San Andreas no sistema convencional foram de $1,05 \mathrm{mS} \mathrm{cm}^{-1}$ e 1,8 $\mathrm{mS} \mathrm{cm}^{1}$, respectivamente, e do sistema orgânico foram de $0,78 \mathrm{mS} \mathrm{cm}^{-1}$ para o cultivar Festival e $0,9 \mathrm{mS} \mathrm{cm}^{-1}$ para o cultivar San Andreas. Revelando, dessa forma, que há influência da variação da $\mathrm{CE}$ no conteúdo de vitamina $\mathrm{C}$.

\section{Conclusões}

Diante dos resultados obtidos, conclui-se que os cultivares Festival e San Andreas, cultivados no sistema convencional, apresentaram teor de vitamina $C$ superior se comparados com os mesmos cultivares cultivados no sistema orgânico. Tais diferenças se devem à maior quantidade de nitrogênio presente na solução nutritiva utilizada no sistema orgânico, uma vez que quanto maior o teor de nitrogênio, menor o teor de vitamina C. Além disso, é possível perceber que a CE é um fator que influencia no teor de vitamina $\mathrm{C}$ em morangos. Po- rém, tais resultados obtidos podem não ser os mesmos de outras pesquisas, uma vez que há muitos fatores que influenciam no teor de vitamina $\mathrm{C}$ nas frutas.

\section{Agradecimentos}

Ao Centro Universitário UNIVATES, ao Laboratório UNIANÁLISES, a CAPES pela bolsa de Pós-Gradução e ao CNPq pela bolsa de Iniciação Científica.

\section{Referências}

Amaro, F. S. (2005). Teores de licopeno e ácido ascórbico em morangos cv. Vila Nova produzidos em sistemas de cultivo orgânico e convencional. 2005. 103 f. Dissertação (Mestrado em Fitotecnia) - Universidade Federal do Rio Grande do Sul, Porto Alegre.

Bom Princípio. (2014).Informações gerais.Disponível em: <http://www.bomprincipio.rs.gov.br/novo/ informacoes.php>. Acesso em: 21 jun 2014.

Brasil. (2007). Ministério da Agricultura. Instrução Normativa n. 17, de 21 de maio de 2007. Brasília.

Cardoso, P. C.; Tomazini, A. P. B.; Stringheta, P. C.; Ribeiro, S. M. R; Pinheiro-Sant'Ana, H. M.. (2011). Vitamin C and carotenoids in organic and conventional fruits grown in Brazil.Food Chemistry, 126, 411-416.

Coultate, T. P. (2004). Alimentos: a química de seus componentes. 3. ed. Artmed.

Cruz-Rus, E.; Amaya, I.; Sánches-Sevilla, J. F.; Botella, M. A.; Valpuesta, V. (2011).Regulation of L-ascorbic acid contend in strawberry fruits. Journal of Experimental Botany, 62 (12), 4191-4201.

Davey, M. W.; Bauw, G.; Montagu, M. V. (1996). Analysis of Ascorbate in plant tissues by HighPerformance Capillary Zone Electrophoresis.Analytical Biochemistry, 8 (19), 8-19.

Godoi, R. dos S. Produtividade e qualidade do morangueiro em sistemas fechados de cultivo sem solo.(2008). 55 f. Dissertação (Mestrado em Agronomia) - Universidade Federal de Santa Maria, Santa Maria.

Lee, K. S.; Kader. A. A. (2000). Preharvest andpostharvestfactors influencing vitamin $C$ content of horticultural crops. Porsthharvest Biology and Technology, 20, 207-220.

Mozafar, A. (1993). Nitrogen fertilizers and the amount of vitamins in plants: a review. Journal of Plant Nutrition, 
16 (12), 2479-2506.

Núcleo de Estudos e Pesquisas em Alimentação. (2011). Tabela brasileira de composição de alimentos. 4 . ed. NEPA-UNICAMP. Disponível em < http:// www.unicamp.br/nepa/taco/contar/taco_4_edicao_ ampliada_e_revisada $>$. Acesso em: 26 ago 2014.

Olsson, M. E.; Ekvall, J.; Gustvasson, K.; Nilsson, J.; Pillai, D.; Sjohlolm, I.; Svensson, U.; Akesson, B.; Nyman, M. G. L. (2004). Antioxidants, low molecular weight carbohydrates, and total antioxidant capacity in strawberries (Fragaria $x$ ananassa): effects of cultivar, ripening, and storage. Journal of Agricultural and Food Chemistry, 52,2490-2498.

Oshita, D.; Jardim, I. C. S. F. (2012).Morango: uma preocupação alimentar, ambiental e sanitária, monitorado por cromatografia líquida moderna. Scientia Chromatographica, 4 (1),52-76.

Penteado, M. de V. C.; Rios, M. D. G.Vitamina C. (2003). In: Penteado, M. de V.C.(Org). Vitaminas: Aspectos nutricionais, bioquímicos, clínicos e analíticos. Manole, 201-225.

Portela, I. P.; Peil, R. M. N.; Rombaldi, C. V. (2012). Efeito da concentração de nutrients no crescimento, produtividade e qualidade de morangos em hidroponia. Horticulturabrasileira, 30 (2), 266-273.

Rice-Evans, C.; Miller, N. J. (1995).Antioxidants - the case for fruit and vegetables in the diet. British Food Journal, 97 (9),35-40.

Rocha, D. A.; Abreu, C. M. P. de; Corrêa, A. D.; Santos, C. D. dos; Fonseca, E. W. N. da. (2008). Análise comparativa de nutrientes funcionais em morangos de diferentes cultivares da região de Lavras/MG. Revista Brasileira de Fruticultura, 30 (4), 1124-1128.

Sanhueza, R. M, V.; Hoffmann, A.; Aantunes, L. E. C.; Freire, J. de M. (2005). Sistema de produção de morango para mesa na região da Serra Gaúcha e Encosta Superior do Nordeste. Embrapa Uva e Vinho, Sistema de Produção, 6 versão eletrônica. Disponível em: http://www.cnpuv.embrapa.br/publica/sprod/ MesaSerraGaucha/index.htm>. Acesso em: 25 mar. 2014.

Silva, V. L. da; Cozzolino, S. M. F. Vitamina C (Ácido Ascórbico). (2007). In: Cpzzolino, S. M. F. (Org.). Biodisponibildade de nutrientes,2. ed. Manole, 305-324. 